Editorial

\title{
Editorial
}

\section{Advances in X-Ray and Inner Shell Processes}

Recent technological developments related to large facilities of third and fourth generation (synchrotron radiation facilities like SOLEIL, and laser facilities like X-FEL) as well as in instrumentation in general allows nowadays measurements with unprecedented resolutions (spatial, temporal, in energy and spin) and counting rates opening the door to new physics. The new theoretical achievements are of crucial importance for the understanding of the processes. Ion collisions and detectors fields are exhibiting impressive new results.

\section{Ions collisions}

Recent progress in intense, high quality ion, photon and electron beams, interacting with well-characterized, high-density, atomic, molecular, clusters or ionic targets in ultra-high vacuum has opened the door to new studies. Relativistic ion-atom collisions lead to X-Rays emission, the polarization of which can now be measured with new kinds of solid-state multi-strip detectors, leading to new insight in ion-atom collisions dynamics. Experimental and theoretical studies on x-ray radiative recombination of multiply charged ions with cooling electrons in storage ring has provided many new high-precision measurements leading to new high-precision tests of fundamental theories like strong-field quantum electro-dynamics. A very active field has developed, in which studies of two photons transitions have greatly improved. A new technique for the measurement of two photon 2E1 transition probabilities has been applied to the de-excitation of the singlet 2 s state of tin and a good agreement of experiment with relativistic calculations has been found. New theoretical calculations are reported for the emission of two-photon by hydrogen-like ions, in the case in which the linear polarization of one of the two photons is measured, providing new ideas for future experiments. A good quantitative understanding of these two-photon transitions has been achieved.

Double ionization of atoms and autoionization of ions

The ratio between double to single 1s photoionization has been measured for light atoms $(\mathrm{Mg}, \mathrm{Al}$, and $\mathrm{Si})$ at the ESRF third generation synchrotron radiation facility. The results are compared to convergent close-coupling calculations for He-like ions. A review on intermediate autoionizing states occurring after electron-ions and photon-ions interactions is presented.

Atoms, clusters and nanoparticles

The issue also contains a review of magnetic dichroism in atomic core level photoemission: laser polarized atoms, complete experiment from magnetic dichroism studies as well as nondipole 
effects in magnetic dichroism. Theoretical and experimental photoemission spectrum of Xe atoms upon photoionization of the Xe $3 d$-subshell has been studied: the $5 p-4 d$ spectrum is emitted in the process of the cascading decay of the $3 d$-vacancy. Electron angular distribution as well as fragmentation dynamics using imaging technique have been investigated on Van der Waals Xenon clusters. Interactions between free clusters and nanoparticles with $\mathrm{X}$-Rays are characterized by photoemission and particle charging as well as elastic light scattering.

\section{Molecules}

It is now feasible to measure multiple (up to to five) electrons in coincidences with high resolution magnetic bottle: a wealth of information about multiple ionization dynamics is thus provided. The electron-ion-ion technique applied to polyatomic molecules allows obtaining information about fragmentation dynamics and symmetries of the core excited states. It was proven that the electron-Ion Vector Correlation method, applied to core ionized $\mathrm{N}_{2} \mathrm{O}$ molecule, gives access to recoil frame photoelectron angular distributions. High Resolution Electron Spectroscopy (HRES) applied to the core ionized nitrogen molecule combined with ab initio predictions revealed oscillations in the $1 \sigma_{g}$ and $1 \sigma_{u}$ photoionization cross sections in a large energy range as predicted by Cohen and Fano. These results are in full agreement with a delocalized picture of the core hole. The study of fully differential cross section of double ionization of $\mathrm{H}_{2}$ in which the four vectors of the four particles are measured or deduced for each ionization event has allowed exhibiting a very interesting phenomenon: the coherent emission of the electron from the two atomic centers of the molecule for particular geometries. Theoretical study on core ionized molecule revealed entanglement between photoelectron and Auger electron.

\section{$X F E L$}

The recent development of high brilliance femtosecond XFEL sources is of extremely high interest. In Hamburg, it is now feasible to obtain X-ray/optical cross correlation through X-ray induced transient optical reflectivity on GaAs. In Stanford, an AMO Instrumentation is under construction at the LCLS X-ray FEL.

\section{Adsorbed molecules and absorption techniques}

Dynamics of adsorbed molecules on surfaces can be probed by core level photoemission technique: using the core hole clock method, it is now possible to probe subfemtosecond electron transfer. Resonant Inelastic X-Ray Scattering is a powerful method (photon in, photon out): bimagnons in antiferromagnetic insulators, $\mathrm{CaCuO}_{2}$ and $\mathrm{BaCuO}_{2}$ have been studied. A recently developed technique: the X-ray Extended Range Technique (XERT) allows high accuracy absolute XAFS measurements.

\section{Linear and circular dichroism}

An application of the generation of short x-ray bursts by X-pinch to astrophysics is highlighted. X-ray photoelectron spectroscopy (XPS) measurements did reveal that Pulsed Electron Deposition (PED) seems an appropriate technique to deposit SiC thin films. X-ray Magnetic Circular Dichroism (XMCD) is a sensitive technique to deposited single molecule magnets (SMMs). XMCD and resonant photoemission are complementary techniques to investigate electronic structures. A recently developed spin-polarized photoemission spectrometer allows determining spin- and angle-resolved photoemission spectra with high energy and momentum resolutions. A theoretical study clearly identified an unknown process: X-ray Magnetic Linear Dichroism (XMLD), which is depending on the orientation of the magnetic moments relative to the crystallographic axes. A new theory allows to simulate high and ultra-high magnetic field behavior of the Ce L2,3 Absorption and XMCD of weakly mixed valence Ce compounds. 


\section{Resonant Inelastic X-Ray Scattering}

The recent progresses of Resonant Inelastic X-Ray Scattering (RIXS) spectrometers in the soft $\mathrm{X}$-ray regime are impressive: it is now possible to well resolve dd excitations allowing quantitative determinations. In the hard X-Ray regime, two dimensional RIXS plane (incident energy emitted energy) is the best way to observe all the electronic transitions. RIXS appears to be a very powerful technique to probe electronic properties of solids under high pressure conditions.

\section{Hard X-ray Photoelectron Spectroscopy}

Hard X-ray Photoelectron Spectroscopy (HAXPES) is receiving a growing interest: the bulk properties can be efficiently probed. Recoil effects are observed for very high kinetic energies after inner shell photoionization of light elements.

\section{Detectors}

A microcalorimeter is an X-ray detector where the deposited heat by a single photon is measured. Newly developed devices cover a broad energy range (from soft to hard X-Rays) with very high resolution. A new method for the measurement of relative conversion efficiencies of high-power, short pulse laser light into $\mathrm{x}$-rays by observing the $\mathrm{x}$-ray radiation of metal alloy foils and other crystalline targets with a curved-crystal transmission spectrometer was presented.

\section{$21^{\text {st }}$ International Conference on X-Ray and Inner Shell Processes}

The present special issue consists of 35 rigorously peer reviewed papers, the topics of which have been selected from material presented at the $21^{\text {st }}$ International Conference on X-Ray and Inner Shell Processes which has been held in Paris between the 22nd and 28th of June 2008. There were ten plenary lectures, twenty invited lectures and thirty-eight progress reports. Three hundred and fifty scientists did attend the X08 conference, belonging to forty-six different countries.

This conference was held during the SOLEIL synchrotron radiation facility first year of operations in which photons have been delivered to users. A visit of SOLEIL during the conference has been organized, which gave to the community the opportunity to be informed of the capabilities of the new facility as well as to have a glimpse of the first exciting results. From the discussion between the XO8 attendees and SOLEIL staff member already a non negligible number of proposals is intended to be submitted for the next call for proposals at SOLEIL.

This conference was the last one of a long series that started by the merging in 1978 of the "X-ray physics" conference, born in 1965, and of the "Inner-shell Ionization" conference born in 1972. It also provided a view of the future that will be build on the merging of this conference series with the VUV series, decided in Berlin in July 2007. The merging of the scientific committees has been finalized during the X2008 conference. The next conference will be organized in July 2010 in Canada by Adam Hitchcock and Stephen Urquhart.

The X2008 conference has been supported by the Ile de France Region, SOLEIL, the International Union of Physics and Applied Physics (IUPAP), the CNRS and the University Pierre and Marie Curie. The conference took place in the historical site of the Cordelier campus of the Pierre and Marie Curie University, at the very center of the "Latin" district, near Odeon, one of the most famous parts of Paris. 